\title{
High ALK mRNA expression has a negative prognostic significance in rhabdomyosarcoma
}

\author{
P Bonvini ${ }^{*}, 1,2,6$, A Zin ${ }^{1,2,6}$, R Alaggio ${ }^{3}$, B Pawel ${ }^{4,5}$, G Bisogno ${ }^{1}$ and A Rosolen ${ }^{1}$ \\ ${ }^{1}$ Pediatric Onco-Haematology Clinic, University-Hospital of Padova, via Giustiniani 3, 35100 Padova, Italy; ${ }^{2}$ Institute of Pediatric \\ Research Città della Speranza, 35100 Padova, Italy; ${ }^{3}$ Department of Pathology, University of Padova, 35100 Padova, Italy; \\ ${ }^{4}$ Department of Pathology, Laboratory Medicine, Children's Hospital of Pennsylvania, Philadelphia, PA 19104, USA and ${ }^{5}$ Perelman \\ School of Medicine at the University of Pennsylvania, Philadelphia, PA 19104, USA
}

Background: Anaplastic lymphoma kinase (ALK) is a receptor tyrosine kinase aberrantly expressed in cancer, but its clinical and functional importance remain controversial. Mutation or amplification of ALK, as well as its expression levels assessed by conventional immunohistochemistry methods, has been linked to prognosis in cancer, although with potential bias because of the semi-quantitative approaches. Herein, we measured ALK mRNA expression in rhabdomyosarcoma (RMS) and determined its clinical impact on patients' stratification and outcome.

Methods: Specimens were obtained from RMS patients and cell lines, and ALK expression was analysed by quantitative RT-PCR, western blotting, IHC, and copy number analysis.

Results: High ALK mRNA expression was detected in the vast majority of PAX3/7-FOXO1-positive tumours, whereas PAX3/7FOXO1-negative RMS displayed considerably lower amounts of both mRNA and protein. Notably, ALK mRNA distinguished unfavourable PAX3/7-FOXO1-positive tumours from PAX3/7-FOXO1-negative RMS $(P<0.0001)$, and also correlated with larger tumour size $(P<0.05)$ and advanced clinical stage $(P<0.01)$, independently of fusion gene status. High ALK mRNA levels were of prognostic relevance by Cox univariate regression analysis and correlated with increased risk of relapse $(P=0.001)$ and survival $(P=0.01)$, whereas by multivariate analysis elevated ALK mRNA expression resulted a negative prognostic marker when clinical stage was not included.

Conclusion: Quantitative assessment of ALK mRNA expression helps to improve risk stratification of RMS patients and identifies tumours with adverse biological characteristics and aggressive behaviour.

Rhabdomyosarcoma (RMS) is the commonest soft tissue malignancy among children and adolescents (Parham and Ellison, 2006), and can be classified into alveolar, embryonal, and the less frequent pleomorphic subtypes (Helman and Meltzer, 2003). More than two-thirds of all RMS are of embryonal histology (ERMS) and tend to occur at younger age, whereas alveolar rhabdomyosarcoma (ARMS) comprises the remaining 20-25\% of cases and is a more aggressive malignancy (Barr et al, 2002). ARMS is characterised by specific chromosomal translocations that give rise to $P A X 3 / 7$ FOXO1 fusion genes, whereas ERMS frequently shows loss of heterozygosity at $11 \mathrm{p} 15$ and a significantly better prognosis
(Barr et al, 2002). Detection of genetic abnormalities is important in the diagnosis of RMS, whereas the identification of potential biomarkers and factors that contribute to tumour growth and progression may lead to more effective risk-adapted therapies and improved outcome (Parham et al, 2007; Davicioni et al, 2009). Receptor tyrosine kinases (RTKs) are among the most interesting therapeutic targets in cancer, because of their involvement in signalling pathways that drive the malignant phenotype and influence proliferation and survival of tumour cells (Manning et al, 2002; Stommel et al, 2007). In RMS, this has been shown for EGFR (erbB1), Her2 (erbB2), IGF1R, c-Met, and PDGFR- $\alpha$, whose

${ }^{\star}$ Correspondence: Dr P Bonvini; E-mail: paolo.bonvini@unipd.it
${ }^{6}$ These authors contributed equally to this work.

Received 7 August 2013; revised 30 September 2013; accepted 1 October 2013; published online 22 October 2013

(C) 2013 Cancer Research UK. All rights reserved 0007-0920/13 
expression varies according to histology, but the consequences of their aberrant activity are similar (Ganti et al, 2006; Rees et al, 2006; Taniguchi et al, 2008; Kim et al, 2009).

Anaplastic lymphoma kinase (ALK) is a RTK belonging to the insulin receptor superfamily that has been reported in RMS, but its role in the pathogenesis of this malignancy is still not known (Pillay et al, 2002). ALK can be detected as a fusion protein kinase or full-length receptor protein in several tumour types (Kelleher and McDermott, 2010), and in both cases it drives tumour biology through phosphorylation and activation of several downstream effector proteins. Inhibition or silencing of ALK leads to cell cycle arrest and apoptosis, supporting the concept that ALK is important for the development and progression of cancer (Barreca et al, 2011). Herein, we analysed ALK mRNA expression, copy number variation, and mutational status in RMS, with clinical and molecular correlation in a cohort of 87 patients, and assessed its activity in vitro. We demonstrated that $A L K$ is upregulated in PAX3/7-FOXO1-positive ARMS, both at mRNA and protein levels, and is capable of signalling when properly stimulated. In these settings, ALK mRNA levels discriminated RMS patients with different outcomes, whereas ALK receptor activity regulated phosphorylation and activation of downstream target proteins. The results of this study may have a direct impact for the ongoing
RMS risk stratification strategies, and major implications for future clinical studies and treatment protocols.

\section{MATERIALS AND METHODS}

Cell lines, primary tumours and normal controls. The cell lines examined in this study were: four PAX-FOXO1-positive (RH30, RH4, RH28, RC2) and one fusion-negative (RH18) ARMS, four ERMS (RD, SMS-CTR, CCA, RH36), three neuroblastoma (NB: LAN5, IMR32 and NB1), and one ALCL (KARPAS-299) cell lines. Cell culture conditions were in accord with the ATCC (Manassas, VA, USA) standard protocols. A total of 87 tissue samples from RMS patients enrolled in the pediatric sarcoma protocols RMS96, RMS4-99 and RMS2005 of the Italian Association of Pediatric Haematology and Oncology (AIEOP) was included after obtaining institutional review board approval. Diagnosis was reviewed by the AIEOP central panel of pathologists in all of the cases and confirmed by RT-PCR using primers for PAX3/7-FOXO1 (ARMS) and MyoD1 (ERMS). Fetal human skeletal muscle total RNA (Stratagene, La Jolla, CA, USA) was used as normal negative control.
A
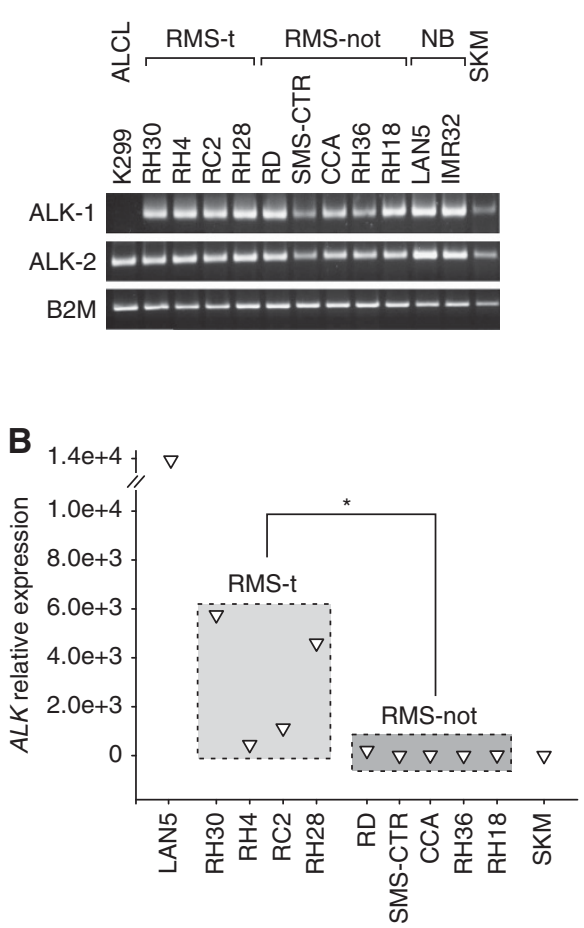

C

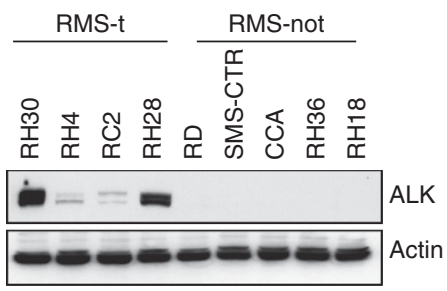

D

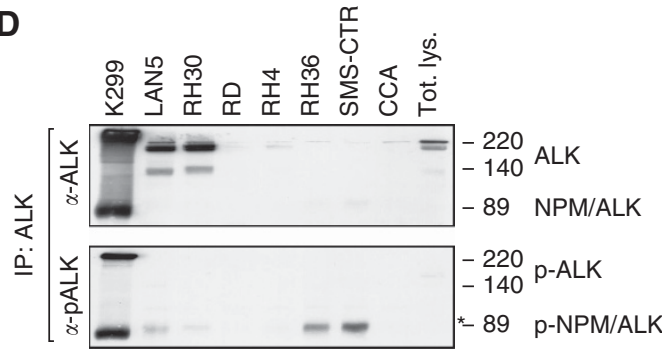

E

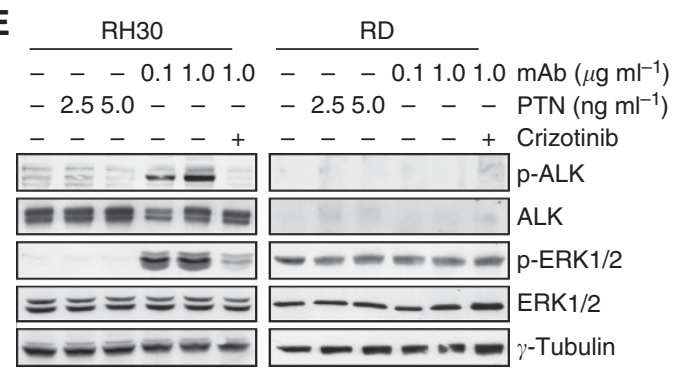

Figure 1. Expression analysis of ALK in RMS cell lines. (A) Expression of ALK mRNA in tumour cell lines, using primers spanning the extracellular region (ALK-1) and the intracytoplasmic portion (ALK-2) of ALK gene. $\beta 2-$ Microglobulin (B2M) was used as housekeeping gene. RMS-t, PAX3/7-FOXO1-positive RMS; RMS-not, PAX3/7-FOXO1-negative RMS; NB, neuroblastoma; SKM, fetal skeletal muscle; ALCL, anaplastic large cell lymphoma. (B) Relative ALK mRNA expression levels in RMS-t and RMS-not cell lines measured by qRT-PCR. LAN5 and SKM were included as positive and negative controls, respectively. Distribution of data is represented by box plot analysis (dashed box). ${ }^{\star} P<0.05$. (C) ALK protein expression in RMS-t and -not cell lines by western blotting, using $\beta$-actin as protein-loading control. (D) Total ( $\alpha$-ALK, upper panel) and phosphorylated ( $\alpha$-pALK, lower panel) ALK proteins detected in RMS-t and RMS-not cell lines after immunoprecipitation (IP:ALK). LAN5 and KARSPAS-299 (K299) cells were used as positive controls for ALK and NPM-ALK expression, respectively. LAN5 total lysates were used as input (Tot.lys.). Asterisk marks non-specific bands in RMS and NB cells. (E) ALK tyrosine phosphorylation in RH30 and RD cell lines exposed to pleiotrophin (PTN) or ALK agonist antibody $(m A b)$ in the presence $(+)$ or absence $(-)$ of ALK inhibitor crizotinib. Total and phosphorylated ALK and ERK1/2 proteins were detected by western blotting. $\gamma$-Tubulin was included as loading control. 
Cell lysis and immunoblotting. Cells in log-phase growth were collected and lysed as previously described (Bonvini et al, 2002), and western blot analysis was performed using standard methods. Antibodies used were: ALK (Invitrogen Corp., Carlsbad, CA, USA); phospho-ALK, ERK1/2 and phospho-ERK1/2 (Cell Signaling Technology, Danvers, MA, USA); $\gamma$-tubulin (Sigma-Aldrich, St Louis, MO, USA); $\beta$-actin (Santa Cruz Biotechnology, Heidelberg, Germany). ALK agonist antibody mAb 16-39 was a generous gift from Professor Tadashi Yamamoto (University of Tokyo, Japan). ALK inhibitor crizotinib was from Selleckchem (Houston, TX, USA). ALK immunoprecipitation was performed using Invitrogen ALK primary antibodies and Protein-G sepharose beads (GE Healthcare Bio-Sciences AB, Stockholm, Sweden) (Bonvini et al, 2002).

DNA, RNA and protein preparation from cell lines and tumour specimens. High-molecular-weight DNA and total RNA were extracted from cell lines using the QIAamp DNA Mini Kit (Qiagen, Milan, Italy) and Trizol reagent (Invitrogen, Milan, Italy), respectively, whereas DNA, RNA and proteins were obtained from human samples by using Trizol reagent (Invitrogen) according to manufacturer's instructions.

RT-PCR and qRT-PCR analysis. PCR amplification of the extracellular (exons 1-3) and intracellular portion (exons 20-22) of $A L K$ was performed as previously described (McDermott et al, 2008), whereas quantitative $A L K$ mRNA expression was determined in cell lines and tumour samples by qRT-PCR using GAPDH (5'-TCCTCTGACTTCAACAGCGA-3' and $5^{\prime}$-GGGTCT TACTCCTTGGAGGC- $3^{\prime}$ ) as normalising reference gene. PCR reactions were performed in triplicate on ABI Prism 7000 Sequence Detection System (Applied Biosystems, Foster City, CA. USA) with SYBR green technology, and the relative expression of $A L K$ was calculated by using the $2^{-\Delta \Delta \mathrm{CT}}$ method.

ALK genomic copy number analysis. Primers and probe were designed to measure the number of genomic copies of $A L K$ in cell lines and in human samples in accordance with Applied Biosystems' TaqMan requirements. ALK primers and probe were 5'-CCCGAGACATCTACAGGTGAGTAA-3', 5' -GGGCTGAGG TGGAAGAGACA- $3^{\prime}$ and $5^{\prime}$-ACTGCCTCACCCCTCCGGGC 3'-FAM labelled, respectively, whereas RNA Pol IID was the endogenous control gene selected to correct for aneuploidy (Williamson et al, 2005).

Immunohistochemistry. ALK expression analysis was performed on formalin-fixed paraffin-embedded RMS specimens, stained with ALK1 antibody (DAKO, Milan, Italy; $1: 50$ ), after deparaffinisation and antigen retrieval. Detection was performed using a biotinylated secondary antibody and a DAB chromogen in haematoxylin counterstained cells (Vectastain ABC Kit Elite, PK-6100, Vector Labs, Burlingame, CA, USA). Each case was considered positive when more than $50 \%$ of the cells had moderate-to-strong cytoplasmic staining.

Statistical analysis. Patients included in the study had a median follow-up of 2.3 years from diagnosis (range 0.02-11.4 years). A receiver-operator characteristic curve (ROC) was calculated to define the cutoff values of $A L K$ mRNA predicting events or fusion gene status with the highest sensitivity and specificity. The areas under the ROC curves (AUCs) were analysed by the Hanley and McNeil method. Survival analysis was performed according to Kaplan-Meier method and differences were calculated by applying log-rank test. Overall survival (OS) was calculated from the date of diagnosis to the date of death for any cause or the last follow-up, whereas progression-free survival (PFS) was calculated from the date of diagnosis to the date of the first event (tumour progression or relapse) or the last follow-up. The association of $A L K$ mRNA expression levels with specific clinical and molecular characteristics was analysed either by Student's $t$-test or Mann-Whitney $U$-test, using SAS statistical programme (SAS-PC, 9.2; SAS Institute Inc., Cary, NC, USA), whereas Cox proportional hazard regression model was applied to evaluate the prognostic potential of $A L K$ mRNA expression regarding PFS and OS of RMS patients. The analysis was performed at both univariate and multivariate levels, with a level of significance defined at a probability value less than $0.05(P<0.05)$ and $0.1(P<0.1)$, respectively.

\section{RESULTS}

Expression and genomic status of ALK in vitro. ALK expression was measured in both ARMS and ERMS cell lines, and compared with that assessed in NB, ALCL, or fetal skeletal muscle cells. PCR detection of both extracellular and cytoplasmic portions of ALK

\begin{tabular}{|c|c|c|c|}
\hline Variable & $\begin{array}{l}\text { ARMS-t } \\
(n=35)\end{array}$ & $\begin{array}{l}\text { ARMS-not } \\
\quad(n=13)\end{array}$ & $\begin{array}{c}\text { ERMS } \\
(n=39)\end{array}$ \\
\hline \multicolumn{4}{|c|}{ Age, years } \\
\hline $\begin{array}{l}\leqslant 10 \\
>10\end{array}$ & $\begin{array}{l}17 \\
18\end{array}$ & $\begin{array}{c}11 \\
2\end{array}$ & $\begin{array}{c}36 \\
3\end{array}$ \\
\hline \multicolumn{4}{|l|}{ Sex } \\
\hline $\begin{array}{l}\text { Male } \\
\text { Female }\end{array}$ & $\begin{array}{l}11 \\
24\end{array}$ & $\begin{array}{l}7 \\
6\end{array}$ & $\begin{array}{l}22 \\
17\end{array}$ \\
\hline
\end{tabular}

\section{Site of disease}

\begin{tabular}{|l|c|c|c}
\hline Parameningeal & 2 & 3 & 10 \\
Orbit & 0 & 1 & 4 \\
Other head and neck & 2 & 2 & 4 \\
Extremity & 18 & 3 & 3 \\
Genitourinary & 1 & 0 & 4 \\
Bladder-prostate & 1 & 0 & 8 \\
Other & 10 & 4 & 6 \\
Unknown & 1 & 0 & 0 \\
\hline
\end{tabular}

\begin{tabular}{|c|c|c|c|}
\hline \multicolumn{4}{|l|}{ Size, cm } \\
\hline$\leqslant 5$ & 8 & 3 & 9 \\
\hline$>5$ & 21 & 9 & 29 \\
\hline Not evaluable & 1 & 0 & 0 \\
\hline Unknown & 5 & 1 & 1 \\
\hline
\end{tabular}

IRS group

\begin{tabular}{|l|c|c|c|}
\hline I & 0 & 0 & 2 \\
II & 2 & 1 & 2 \\
III & 10 & 9 & 30 \\
IV & 23 & 3 & 4 \\
Unknown & 0 & 0 & 1 \\
\hline
\end{tabular}

Evaluable ALK

\begin{tabular}{|l|c|c|c|}
\hline qRT-PCR & 35 & 13 & 39 \\
GCN & 16 & 6 & 20 \\
\hline
\end{tabular}

Survival analysis

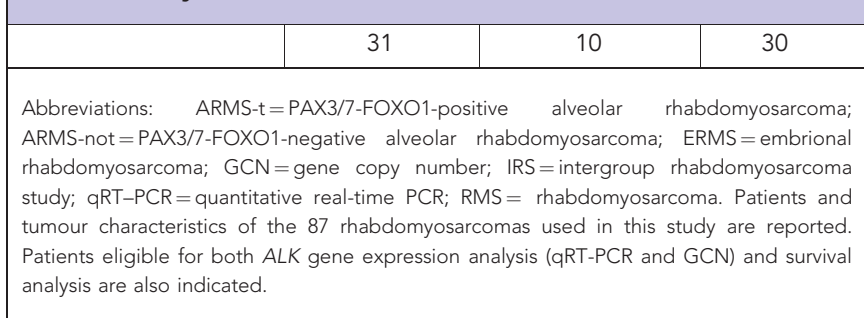



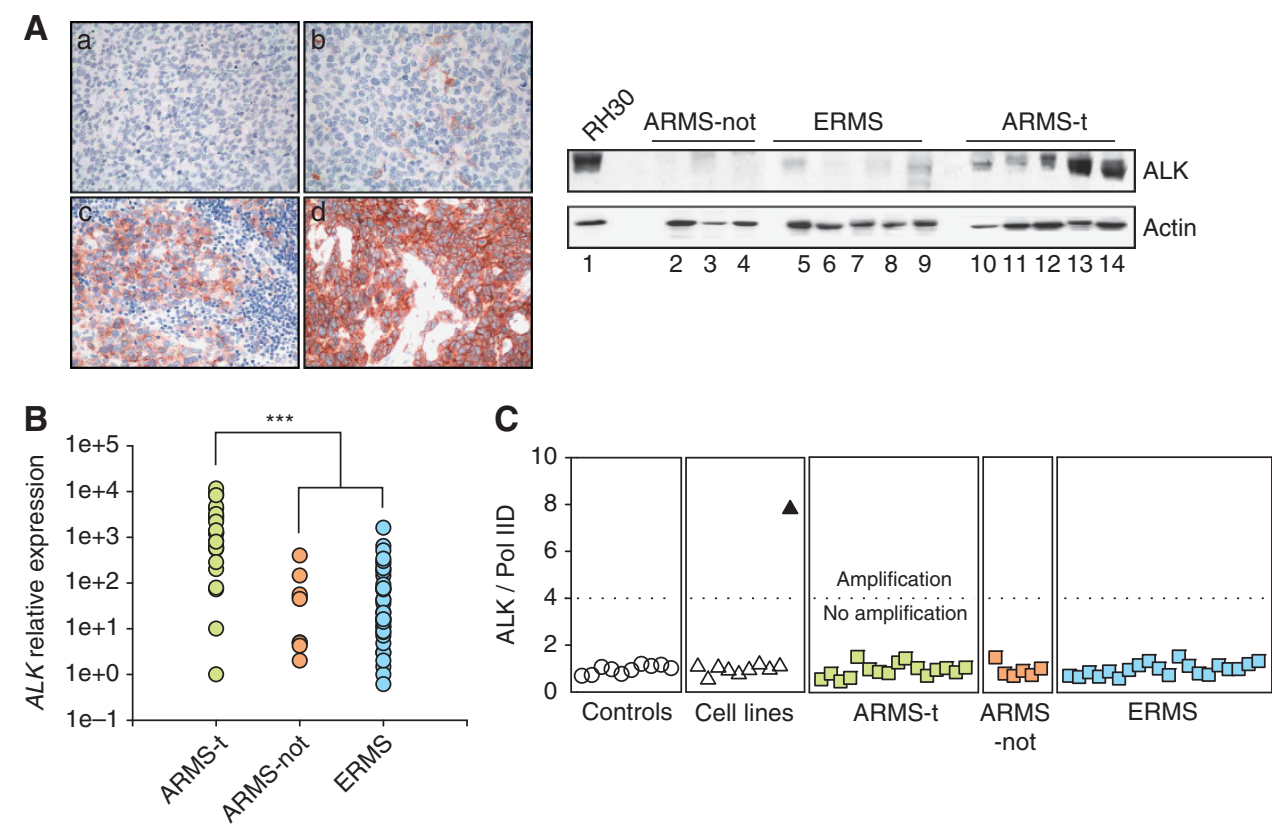

Figure 2. ALK mRNA and protein expression in RMS tumour specimens. (A) ALK protein expression analysis in PAX3/7-FOXO1-negative (ARMSnot, a; ERMS, b) and -positive (ARMS-t, $c$ and d) RMS tumours, by immunohistochemistry (left) and western blotting (right). (B) Point plot analysis of ALK mRNA relative expression in PAX3/7-FOXO1-positive (ARMS-t) and -negative (ARMS-not) ARMS, and in ERMS tumours by qRT-PCR. P-values were calculated using the Student's t-test. ${ }^{\star \star \star} P<0.001$. (C) ALK gene amplification analysis in RMS tumours and cell lines, normalised for RNA Polymerase IID expression (Pol IID). Graph shows true ALK amplification in normal cells (open circles), tumour cell lines (RMS, open triangles; NB1, closed triangle), and RMS specimens (closed squares). Dotted line represents cutoff for definition of gene amplification (gene copy number $\geqslant 4$ ).

was carried out using different set of primers, in order to discriminate the expression of full-length ALK and truncated variants arising from chromosomal rearrangements, whereas western blot analysis was performed to determine endogenous protein expression and activity. We found that all RMS and NB cell lines expressed both the extracellular and cytoplasmic portions of ALK, whereas ALCL cells KARPAS-299 exhibited only the cytoplasmic portion of ALK as a result of variant NPM-ALK expression (Figure 1A). ALK mRNA was quantified by qRT-PCR, and its relative expression compared in PAX3/7-FOXO1-positive (RMS-t) and -negative (RMS-not) cell lines. ALK mRNA levels were significantly higher in ARMS cell lines expressing PAX3/7FOXO1 (RMS-t) compared with ERMS (Figure $1 \mathrm{~B}, P=0.032$ ), and this correlated with higher ALK protein expression (Figure 1C, RMS-t vs RMS-not). However, when ALK was immunoprecipitated and probed with phosphospecific antibodies, no basal receptor phosphorylation, like that of NPM-ALK in ALCL cells, was observed (Figure 1D, lower panel), suggesting that ALK is not constitutively activated in these cells. To rule out any defect of RTK activity, we also conducted a mutagenesis screen in search of mutations in the $A L K$ gene, and we analysed inducible ALK phosphorylation upon stimulation with putative ligand pleiotrophin (PTN) or ALK agonist monoclonal antibody mAb16-39. No point mutations were detected in the tyrosine kinase domain of ALK (exons 22-25, data not shown), and PTN failed to activate ALK when added to the cells (Figure 1E). In contrast, exposing PAX3/7-FOXO1-postive RH30 cells to mAb16-39 resulted in a dose-dependent increase of ALK phosphorylation, which was accompanied by the phosphorylation of downstream ERK1/2 kinase and completely prevented by the dual ALK-MET inhibitor crizotinib (Figure 1E). In contrast, neither mAb16-39 nor crizotinib changed basal ERK1/2 phosphorylation in ALK-negative RD ERMS cells.

ALK mRNA expression in RMS tumours. The expression of ALK was then measured in RMS human specimens (Table 1).

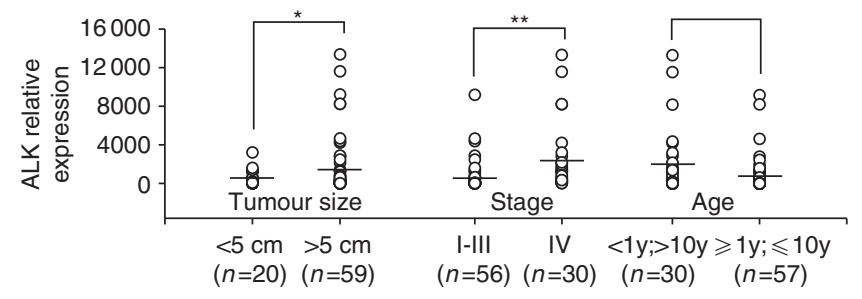

Figure 3. Correlation between ALK mRNA expression levels and clinicopathological parameters in RMS tumours. ALK mRNA levels, assessed by qRT-PCR and normalised to GAPDH housekeeping gene, were compared among groups classified by tumour size, staging, or age. ${ }^{\star} P<0.05 ;{ }^{\star *} P<0.01$.

Consistent with our previous findings, ALK protein was barely detectable in PAX3/7-FOXO1-negative RMS, whereas it showed moderate-to-highly expression in PAX3/7-FOXO1-positive ARMS (Figure 2A). Quantitative ALK mRNA expression analysis confirmed that PAX3/7-FOXO1-positive ARMS tumours (ARMS-t) displayed higher ALK mRNA levels than fusion genenegative RMS, with median expression values of 1405 ( $\mathrm{min} .=10$ / $\max .=13370$ units) $v s 23$ ( $\mathrm{min} .=1 / \max .=1614$ units), respectively. This difference was statistically significant (Figure 2B, $P<0.001$ ), whereas it was not when $A L K$ mRNA median expression levels of ARMS-not and ERMS were compared $(P=0.79)$. Because DNA amplification can lead to increased mRNA expression, we also examined $A L K$ DNA copy number for a set of RMS specimens (ARMS- $t=16$; ARMS- $n$ ot $=6$; ERMS $=20)$ and cell lines $(n=9)$, and compared with that measured in healthy individuals $(n=10)$ or in cancer cells known for true $A L K$ amplification (NB1). The results reported in Figure $2 \mathrm{C}$ demonstrated no obvious $A L K$ gene amplification in RMS samples, when corrected for aneuploidy with the RNA Pol IID gene (Figure 2C, RMS median ALK/Pol IID =1; NB1 ALK/Pol $\mathrm{IID}=8$ ), suggesting an altered regulation of $A L K$ at the gene 

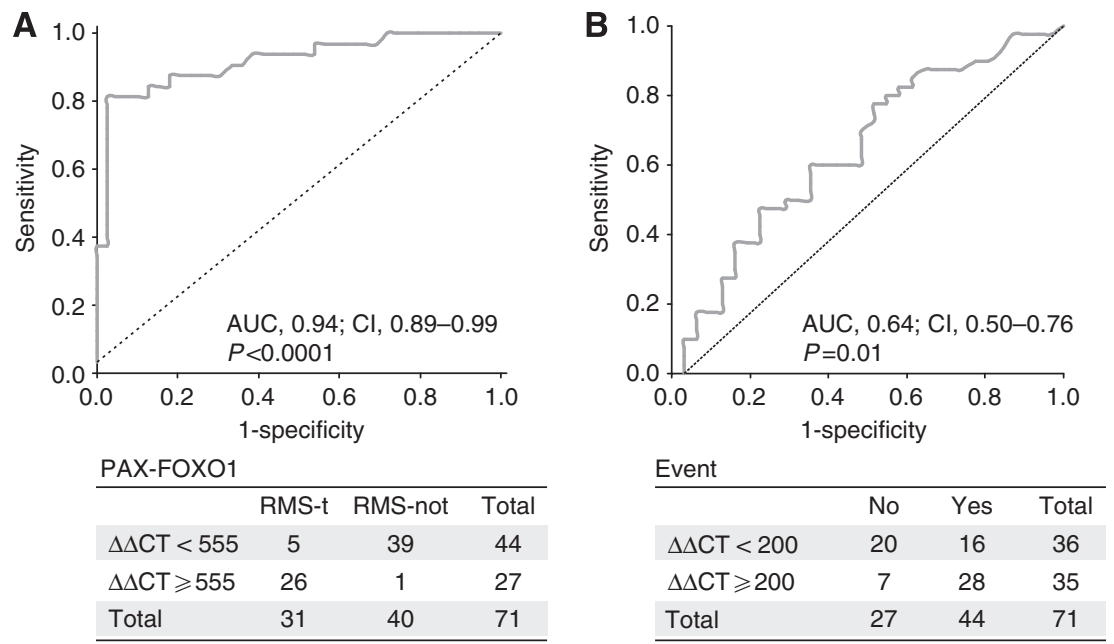

Figure 4. Receiver operating characteristic curve (ROC) analysis. ROCs showing the sensitivity and specificity of ALK mRNA as a parameter to classify RMS patients $(n=71)$ on the basis of the fusion gene status (PAX-FOXO1, RMS-t vs RMS-not; $\Delta \Delta C T=555)(\mathbf{A})$ or the risk of failure (EVENT, $N O$ vs YES; $\Delta \Delta C T=200$ ) (B). P-values, AUC, and 95\% Cls were computed by using SAS statistical programme. Abbreviations: AUC $=$ area under the curve; $\mathrm{Cl}$, confidence interval.

promoter rather than at its genomic level. In addition, consistent with previous studies that demonstrated that ALK point mutations are uncommon in RMS cells (van Gaal et al, 2011; Yoshida et al, 2013), we found no significant variations in the ALK tyrosine kinase domain when bidirectional sequencing was performed (data not shown).

Prognostic implications of $A L K$ mRNA expression. To investigate a possible correlation between high $A L K$ mRNA expression and RMS patient outcome, we performed association analysis with known RMS prognostic factors, such as initial tumour size, clinical stage, and age at diagnosis. Data analysis of RMS patients showed a positive correlation between high $A L K$ mRNA levels and advanced stage of disease (Stage IV, $P=0.01)$ or tumour size $(>5 \mathrm{~cm}$, $P=0.04)$, whereas ALK mRNA expression and age at diagnosis were not significantly associated ( $<1$ to $>10 \mathrm{y}, P=0.06$; Figure 3 ). In addition, ROC curve analysis was carried out in RMS patients for which clinical follow-up was available, taking into account the fusion gene status (PAX3/7-FOXO1 expression) and patients' survival. We demonstrated that $\Delta \Delta \mathrm{CT}=555$ was the ALK mRNA cutoff level capable of distinguishing with extremely high accuracy PAX3/7FOXO1-positive RMS from fusion gene-negative tumours (Figure 4A, AUC $=0.94 ; 95 \% \mathrm{CI}=0.89-0.99, P<0.0001$ ), whereas $\Delta \Delta \mathrm{CT}=200$ was the discriminating value that best characterised patients according to risk of relapse (Figure 4B, AUC $=0.64 ; 95 \%$ $\mathrm{CI}=0.50-0.76 ; P=0.01)$. Indeed, RMS patients with low $A L K$ mRNA levels $(\Delta \Delta C T<200)$ had a significant reduced risk of failure compared with those with high ALK mRNA expression $(\Delta \Delta \mathrm{CT}>200 ; 44 \%$ vs $80 \%$, respectively), whereas tumours with very high $A L K$ transcript levels $(\Delta \Delta \mathrm{CT}>555)$ were almost exclusively PAX3/7-FOXO1-positive RMS (RMS-t = 84\%; RMSnot $=2.5 \%$ ). The prognostic value of the quantitative $A L K$ mRNA assessment was substantiated by Kaplan-Meier analysis for PFS and OS of RMS patients distinguished based on the specific quantitative $A L K$ mRNA cutoff value $\triangle \triangle C T=200$, which demonstrated a significantly poorer prognosis of patients with high $A L K$ mRNA levels $(A L K$ high $=\Delta \Delta \mathrm{CT}>200)$, irrespective of tumour histology and fusion-gene status (Figure 5, log-rank test, PFS $P=0.001$, OS $P=0.01)$. Cox regression analysis confirmed these data, in particular when $A L K$ mRNA was included together with known RMS risk factors. In our cohort of 71 patients, clinical stage (Stage IV), histology (ARMS), fusion gene status (PAX3/7-FOXO1-positive), and ALK mRNA $(\Delta \Delta C T>200)$ were all significantly associated with lower survival, whereas age and tumour size at diagnosis were not
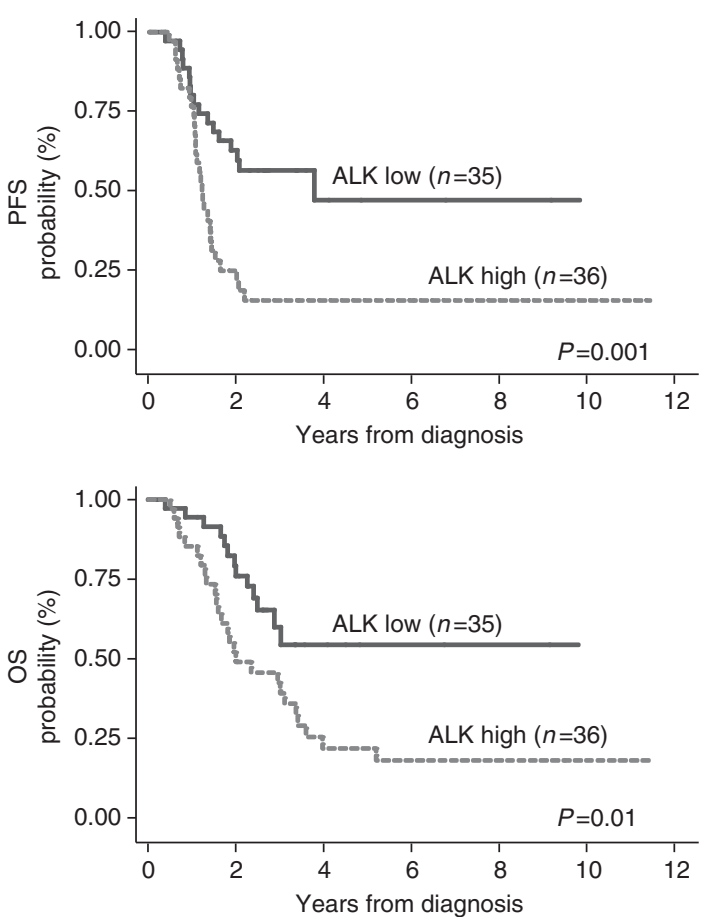

Figure 5. Prognostic significance of ALK gene expression. KaplanMeier and log-rank analysis for progression-free (PFS) and overall survival (OS) of RMS patients ( $n=71$ ) based on specific quantitative ALK mRNA cutoff value ( $A L K$ low $=\Delta \Delta C T<200 ; A L K$ high $=\Delta \Delta C T \geqslant 200)$ identify two subgroups of patients with significantly different outcome (PFS, $P=0.001 ;$ OS, $P=0.01$ ).

(Table 2). ALK mRNA was a significant negative predictor of both PFS $(P=0.001)$ and OS $(P=0.013)$ in univariate analysis; whereas in multivariate analysis, RMS clinical stage was a stronger prognostic factor (Stage, OS: $P<0.0001, \mathrm{HR}=3.8,95 \% \mathrm{CI}=2.0-7.3$; PFS: $P=0.0004, \mathrm{HR}=3.0,95 \% \mathrm{CI}=1.6-5.5)$. However, high $A L K$ mRNA level was predictive on increased risk of relapse in multivariate analysis when tumour stage was not considered ( $A L K$ $\Delta \Delta \mathrm{CT} \geqslant 200$, PFS: $P=0.0017, \mathrm{HR}=2.7,95 \% \mathrm{CI}=1.5-5.1)$, as it was ARMS tumour histology in a model based on patient OS. Of note, ALK protein expression has been reported to correlate with ARMS histology, metastasis at presentation, and advanced clinical 


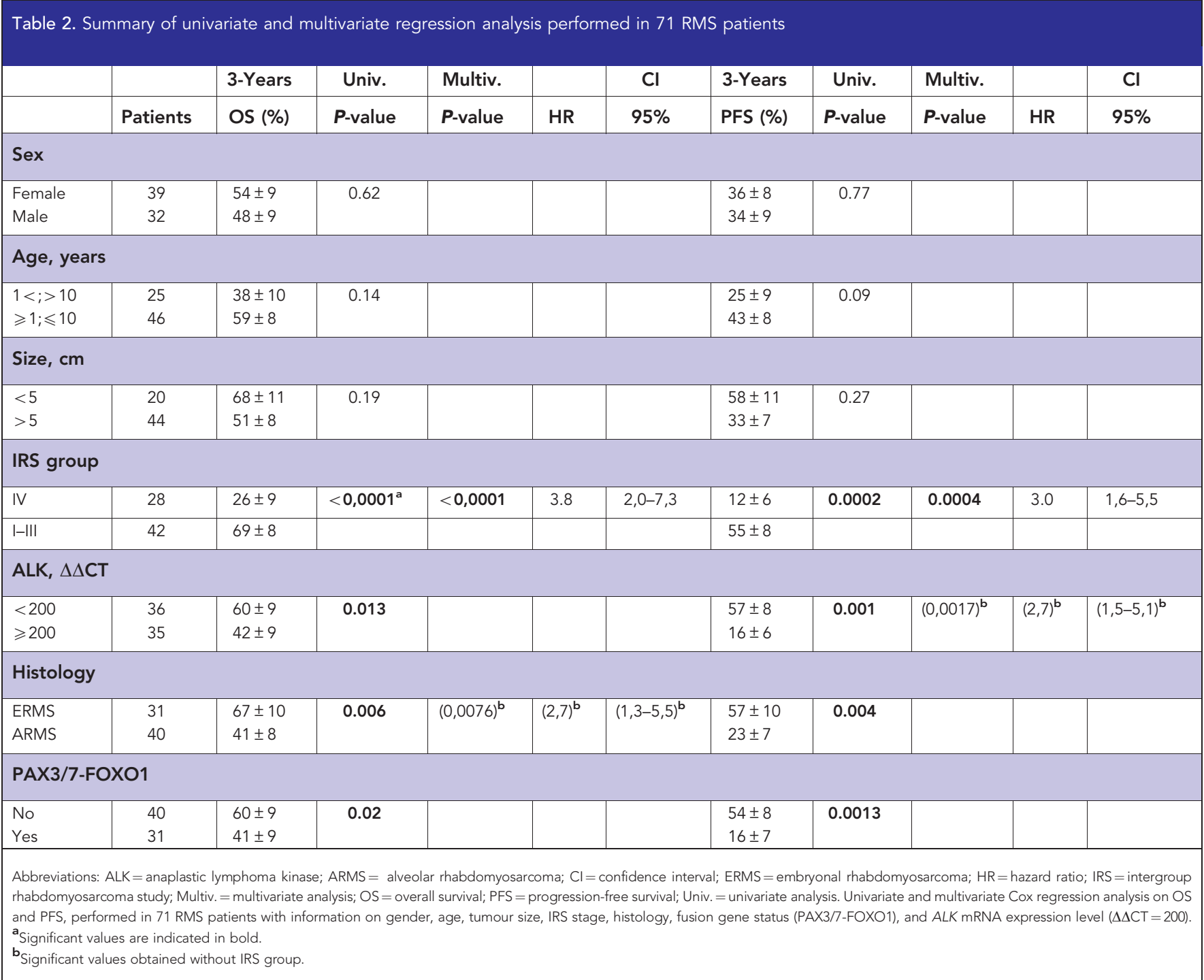

stage, but not with increased risk of relapse and death of RMS patients (van Gaal et al, 2011; Yoshida et al, 2013).

\section{DISCUSSION}

In this study, we examined the mRNA expression of $A L K$, a novel PAX3/FOXO1 target gene (Cao et al, 2010), in pediatric RMS tumours and cell lines, to discern the correlation with the clinical phenotype and establish the impact on patients' risk assessment. ALK is a RTK that can be expressed both as truncated fusion protein and full-length receptor kinase (Motegi et al, 2004; Soda et al, 2007), and it may induce malignant transformation both in vivo and in vitro. ALK overexpression correlates with advanced clinical stage and poor prognosis in several tumour types, and its inhibition results in a marked decrease of cell growth and survival (Mosse et al, 2008; Schulte et al, 2011). To date, several studies have reported on the protein expression of ALK in RMS and clinicopathological analysis using immunohistochemistry (Pillay et al, 2002; Corao et al, 2009; Yoshida et al, 2013). However, because of the different detection frequency and sensitivity observed, the clinical and functional importance of ALK in RMS remain uncertain yet.

Herein, we demonstrated that ALK expression varies broadly in RMS, with mRNA and protein levels significantly higher in PAX3/7-FOXO1-positive ARMS tumours and cell lines (Corao et al, 2009) compared with both ERMS and PAX3/7-FOXO1negative ARMS (RMS-not). All the previous studies have used semi-quantitative methodologies to assess the expression of ALK in RMS, and according to these data, ALK protein expression was shown to be different in and between RMS subgroups (Corao et al, 2009; van Gaal et al, 2011; Yoshida et al, 2013). Herein, we provided the first evidence that ALK mRNA level, measured by quantitative RT-PCR, is a suitable marker for RMS patients stratification, as it is significantly higher in RMS tumours characterised by adverse clinicopathological parameters, such as the unfavourable PAX3/7-FOXO1-postive histology, advanced stage of disease, and larger tumour size at diagnosis, and also correlates with patients' increased risk of relapse and lower survival. In these settings, high $A L K$ mRNA levels identified patients with higher risk of failure and poor outcome, and distinguished with high accuracy PAX3/7-FOXO1-positive ARMS and aggressive PAX3/7-FOXO1-negative tumours. Our findings are in accordance with metagene analysis performed by Williamson et al, which demonstrated that ALK gene expression highly discriminates PAX3/7-FOXO1-positive ARMS from fusion gene-negative RMS tumours (Williamson et al, 2010), but also with the observation that ALK protein may be predictive of poor patient survival in ARMS and metastatic ERMS (van Gaal et al, 2011; Lee et al, 2013). Indeed, we observed a significant trend towards a worse outcome of PAX3/7-FOXO1-negative RMS patients with high $A L K$ mRNA levels, both when these patients 
were defined according to the $A L K$ median expression level and pre-specified cutoff values. Moreover, in Cox univariate regression analysis, high $A L K$ mRNA levels $(\Delta \Delta \mathrm{CT}>200)$ predicted a decreased PFS and OS independently of PAX3/7-FOXO1 expression, and Kaplan-Meier survival curves substantiated these conclusions. Although $A L K$ mRNA did not turn out to be an independent prognostic marker in multivariate analysis considering RMS clinical stage, tumour size at diagnosis, age, and fusion gene status as risk factors, it had an unfavourable prognostic value when multivariate analysis was performed without considering tumour staging, supporting the concept that high ALK mRNA expression may be predictive of an unfavourable phenotype in primary RMS. Consistent with our results, ALK overexpression in NB predicts lower patient survival in a univariate Cox regression model using ALK mRNA as a continuous variable, whereas in a multivariate regression model, tumour stage, age, and $1 \mathrm{p}$ status result stronger independent negative variables (Schulte et al, 2011).

High ALK mRNA expression levels in RMS, however, were not due to specific ALK copy number gain or mutational events, because true ALK amplification or point mutations were not detected in both tumours and cell lines. In this study, we used a quantitative approach to detect true $A L K$ amplification, and RNA Polymerase IID (RNA Pol IID) gene to correct for aneuploidy. No obvious amplification of the $A L K$ gene was observed, whereas all the single substitutions detected after bidirectional sequencing of the $A L K$ tyrosine kinase domain were synonymous or silent mutations. Consistently, true ALK amplification is rather uncommon in RMS and other tumour types (i.e., NB), either when it is measured by FISH or qRT-PCR (Schulte et al, 2011; Fleuren et al, 2013; Yoshida et al, 2013).

Finally, the strong correlation between $A L K$ mRNA expression and patient outcome, together with the observation that ALK is capable of signalling in RMS cells, suggests that ALK is a RTK noteworthy for further investigation in this malignancy. To date, this is the first study that achieved inducible ALK phosphorylation in RMS cells in vitro, examined its activation and inhibited its activity. Although the role of ALK in RMS cells is still under investigation, we provided evidences that ALK may affect RMS cell signalling and be a potential target for small-molecule inhibitors in these cells.

In conclusion, this is the first study that implicates $A L K$ mRNA as a negative factor in RMS and demonstrates that the quantitative assessment of ALK mRNA expression levels may be used to improve risk stratification of RMS patients independently of fusion gene status and tumour histology. These findings, although preliminary, warrant consideration for future treatment strategies of this malignancy.

\section{ACKNOWLEDGEMENTS}

We thank Professor Tadashi Yamamoto for kindly providing ALK agonist antibody mAb16-39; Dr G Franceschetto and Dr E Carraro for the statistical analysis; Dr I Zanetti for data analysis and interpretation; E Tosato for her technical expertise and support. This work was supported by Fondazione Città della Speranza, MIUR (Ministero Istruzione Università e Ricerca), and by AIRC (Associazione Italiana per la Ricerca sul Cancro).

\section{CONFLICT OF INTEREST}

The authors declare no conflict of interest.

\section{REFERENCES}

Barr FG, Qualman SJ, Macris MH, Melnyk N, Lawlor ER, Strzelecki DM, Triche TJ, Bridge JA, Sorensen PH (2002) Genetic heterogeneity in the alveolar rhabdomyosarcoma subset without typical gene fusions. Cancer Res 62: 4704-4710.

Barreca A, Lasorsa E, Riera L, Machiorlatti R, Piva R, Ponzoni M, Kwee I, Bertoni F, Piccaluga PP, Pileri SA, Inghirami G (2011) Anaplastic lymphoma kinase in human cancer. J Mol Endocrinol 47: R11-R23.

Bonvini P, Gastaldi T, Falini B, Rosolen A (2002) Nucleophosmin-anaplastic lymphoma kinase (NPM-ALK), a novel Hsp90-client tyrosine kinase: down-regulation of NPM-ALK expression and tyrosine phosphorylation in $\operatorname{ALK}(+) \operatorname{CD} 30(+)$ lymphoma cells by the Hsp90 antagonist 17-allylamino,17-demethoxygeldanamycin. Cancer Res 62: 1559-1566.

Cao L, Yu Y, Bilke S, Walker RL, Mayeenuddin LH, Azorsa DO, Yang F, Pineda M, Helman LJ, Meltzer PS (2010) Genome-wide identification of PAX3-FKHR binding sites in rhabdomyosarcoma reveals candidate target genes important for development and cancer. Cancer Res 70: 6497-6508.

Corao DA, Biegel JA, Coffin CM, Barr FG, Wainwright LM, Ernst LM, Choi JK, Zhang PJ, Pawel BR (2009) ALK expression in rhabdomyosarcomas: correlation with histologic subtype and fusion status. Pediatr Dev Pathol 12: $275-283$.

Davicioni E, Anderson MJ, Finckenstein FG, Lynch JC, Qualman SJ, Shimada H, Schofield DE, Buckley JD, Meyer WH, Sorensen PH, Triche TJ (2009) Molecular classification of rhabdomyosarcoma-genotypic and phenotypic determinants of diagnosis: a report from the Children's Oncology Group. Am J Pathol 174: 550-564.

Fleuren ED, Roeffen MH, Leenders WP, Flucke UE, Vlenterie M, Schreuder HW, Boerman OC, van der Graaf WT, Versleijen-Jonkers YM (2013) Expression and clinical relevance of MET and ALK in Ewing sarcomas. Int J Cancer 133: $427-436$.

Ganti R, Skapek SX, Zhang J, Fuller CE, Wu J, Billups CA, Breitfeld PP, Dalton JD, Meyer WH, Khoury JD (2006) Expression and genomic status of EGFR and ErbB-2 in alveolar and embryonal rhabdomyosarcoma. Mod Pathol 19: 1213-1220.

Helman LJ, Meltzer P (2003) Mechanisms of sarcoma development. Nat Rev Cancer 3: 685-694.

Kelleher FC, McDermott R (2010) The emerging pathogenic and therapeutic importance of the anaplastic lymphoma kinase gene. Eur J Cancer 46: 2357-2368.

Kim SY, Toretsky JA, Scher D, Helman LJ (2009) The role of IGF-1R in pediatric malignancies. Oncologist 14: 83-91.

Lee JS, Lim SM, Rha SY, Roh JK, Cho YJ, Shin KH, Yang WI, Kim SH, Kim HS (2013) Prognostic implications of anaplastic lymphoma kinase gene aberrations in rhabdomyosarcoma; an immunohistochemical and fluorescence in situ hybridisation study. J Clin Pathol; e-pub ahead of print 6 August 2013; doi:10.1136/jclinpath-2013-201655.

Manning G, Whyte DB, Martinez R, Hunter T, Sudarsanam S (2002) The protein kinase complement of the human genome. Science $\mathbf{2 9 8}$ 1912-1934.

McDermott U, Iafrate AJ, Gray NS, Shioda T, Classon M, Maheswaran S, Zhou W, Choi HG, Smith SL, Dowell L, Ulkus LE, Kuhlmann G, Greninger P, Christensen JG, Haber DA, Settleman J (2008) Genomic alterations of anaplastic lymphoma kinase may sensitize tumors to anaplastic lymphoma kinase inhibitors. Cancer Res 68: 3389-3395.

Mosse YP, Laudenslager M, Longo L, Cole KA, Wood A, Attiyeh EF, Laquaglia MJ, Sennett R, Lynch JE, Perri P, Laureys G, Speleman F, Kim C, Hou C, Hakonarson H, Torkamani A, Schork NJ, Brodeur GM, Tonini GP, Rappaport E, Devoto M, Maris JM (2008) Identification of ALK as a major familial neuroblastoma predisposition gene. Nature 455: 930-935.

Motegi A, Fujimoto J, Kotani M, Sakuraba H, Yamamoto T (2004) ALK receptor tyrosine kinase promotes cell growth and neurite outgrowth. J Cell Sci 117: 3319-3329.

Parham DM, Ellison DA (2006) Rhabdomyosarcomas in adults and children: an update. Arch Pathol Lab Med 130: 1454-1465.

Parham DM, Qualman SJ, Teot L, Barr FG, Morotti R, Sorensen PH, Triche TJ, Meyer WH (2007) Correlation between histology and PAX/FKHR fusion status in alveolar rhabdomyosarcoma: a report from the Children's Oncology Group. Am J Surg Pathol 31: 895-901.

Pillay K, Govender D, Chetty R (2002) ALK protein expression in rhabdomyosarcomas. Histopathology 41: 461-467. 
Rees H, Williamson D, Papanastasiou A, Jina N, Nabarro S, Shipley J, Anderson J (2006) The MET receptor tyrosine kinase contributes to invasive tumour growth in rhabdomyosarcomas. Growth Factors 24: 197-208.

Schulte JH, Bachmann HS, Brockmeyer B, Depreter K, Oberthur A, Ackermann S, Kahlert Y, Pajtler K, Theissen J, Westermann F, Vandesompele J, Speleman F, Berthold F, Eggert A, Brors B, Hero B, Schramm A, Fischer M (2011) High ALK receptor tyrosine kinase expression supersedes ALK mutation as a determining factor of an unfavorable phenotype in primary neuroblastoma. Clin Cancer Res 17: 5082-5092.

Soda M, Choi YL, Enomoto M, Takada S, Yamashita Y, Ishikawa S, Fujiwara S, Watanabe H, Kurashina K, Hatanaka H, Bando M, Ohno S, Ishikawa Y, Aburatani H, Niki T, Sohara Y, Sugiyama Y, Mano H (2007) Identification of the transforming EML4-ALK fusion gene in non-small-cell lung cancer. Nature 448: 561-566.

Stommel JM, Kimmelman AC, Ying H, Nabioullin R, Ponugoti AH, Wiedemeyer R, Stegh AH, Bradner JE, Ligon KL, Brennan C, Chin L, DePinho RA (2007) Coactivation of receptor tyrosine kinases affects the response of tumor cells to targeted therapies. Science 318: 287-290.

Taniguchi E, Nishijo K, McCleish AT, Michalek JE, Grayson MH, Infante AJ, Abboud HE, Legallo RD, Qualman SJ, Rubin BP, Keller C (2008) PDGFR-A is a therapeutic target in alveolar rhabdomyosarcoma. Oncogene 27: 6550-6560. van Gaal JC, Flucke UE, Roeffen MH, de Bont ES, Sleijfer S, MavinkurveGroothuis AM, Suurmeijer AJ, van der Graaf WT, Versleijen-Jonkers YM (2011) Anaplastic lymphoma kinase aberrations in rhabdomyosarcoma: clinical and prognostic implications. J Clin Oncol 30: 308-315.

Williamson D, Lu YJ, Gordon T, Sciot R, Kelsey A, Fisher C, Poremba C, Anderson J, Pritchard-Jones K, Shipley J (2005) Relationship between MYCN copy number and expression in rhabdomyosarcomas and correlation with adverse prognosis in the alveolar subtype. J Clin Oncol 23: $880-888$.

Williamson D, Missiaglia E, de Reynies A, Pierron G, Thuille B, Palenzuela G, Thway K, Orbach D, Lae M, Freneaux P, Pritchard-Jones K, Oberlin O, Shipley J, Delattre O (2010) Fusion gene-negative alveolar rhabdomyosarcoma is clinically and molecularly indistinguishable from embryonal rhabdomyosarcoma. J Clin Oncol 28: 2151-2158.

Yoshida A, Shibata T, Wakai S, Ushiku T, Tsuta K, Fukayama M, Makimoto A, Furuta K, Tsuda H (2013) Anaplastic lymphoma kinase status in rhabdomyosarcomas. Mod Pathol 26: 772-781.

This work is published under the standard license to publish agreement. After 12 months the work will become freely available and the license terms will switch to a Creative Commons AttributionNonCommercial-Share Alike 3.0 Unported License. 